\title{
The synergistic action of cyclodextrin-based adsorbent and advanced oxidation processes for sulfamethoxazole removal from water
}

\author{
V. Rizzi ${ }^{1} \cdot$ R. Romita ${ }^{1}$ - V. M. Gómez-López ${ }^{2} \cdot$ J. Gubitosa $^{1}$ - J. A. Gabaldón ${ }^{2} \cdot$ M. I. Fortea Gorbe ${ }^{2} \cdot$ T. Gómez-Morte ${ }^{2}$. \\ P. Cosma ${ }^{1} \cdot$ P. Fini ${ }^{3}$ (I)
}

Received: 4 June 2021 / Revised: 15 September 2021 / Accepted: 28 December 2021 / Published online: 29 January 2022

(c) The Author(s) 2022

\begin{abstract}
In this work, the removal of sulfamethoxazole from water was studied by adsorption on a cyclodextrin-epichlorohydrin copolymer and through Advanced Oxidation Processes. The adsorption was efficient and fast, requiring only 10 min to reach the equilibrium; it was also highly favored, with adsorption efficiency higher than $80 \%$, at slightly acidic $\mathrm{pH}$ and at room temperature. The desorption of sulfamethoxazole was obtained by raising the temperature to $80^{\circ} \mathrm{C}$. The regenerated polymer has been reused multiple times without any loss in performance, allowing a reduction in costs associated with the process. As an alternative method to regenerate the adsorbent material, the photodegradation of sulfamethoxazole adsorbed on the polymer alone and in the presence of $\mathrm{TiO}_{2}$ as a catalyst through continuous irradiation with UV light and Pulsed Light was attempted and studied. For this purpose, polymeric adsorbents containing different amounts of $\mathrm{TiO}_{2}$ were synthesized and tested. The Pulsed Light showed the highest efficiency since it allowed the complete removal of sulfamethoxazole, regardless of the presence of $\mathrm{TiO}_{2}$ which, however, reduces adsorption efficiency of the material. Furthermore, a qualitative assessment of formed pulsed light by-products was also attempted. The remains of SMX in solution were decomposed by Pulsed Light technology and the effect of the presence of hydrogen peroxide on the photodegradation process was also studied. Despite the presence of by-products after Advanced Oxidation Processes, the procedure proposed in this work is effective and easy to apply to water treatment plants, thanks to the use of environmental-friendly adsorbents and light sources.
\end{abstract}

Editorial responsibility: Samareh Mirkia.

P. Fini

p.fini@ba.ipcf.cnr.it

1 Dip. Chimica, Università Degli Studi Di Bari "Aldo Moro", Via Orabona, 4, 70126 Bari, Italy

2 Molecular Recognition and Encapsulation Research Group (REM), Health Sciences Department, Universidad Católica de Murcia (UCAM), Campus de los Jerónimos 135, 30107 Guadalupe, Spain

3 Consiglio Nazionale Delle Ricerche CNR-IPCF, SS Bari, Via Orabona, 4, 70126 Bari, Italy 


\section{Graphical abstract}

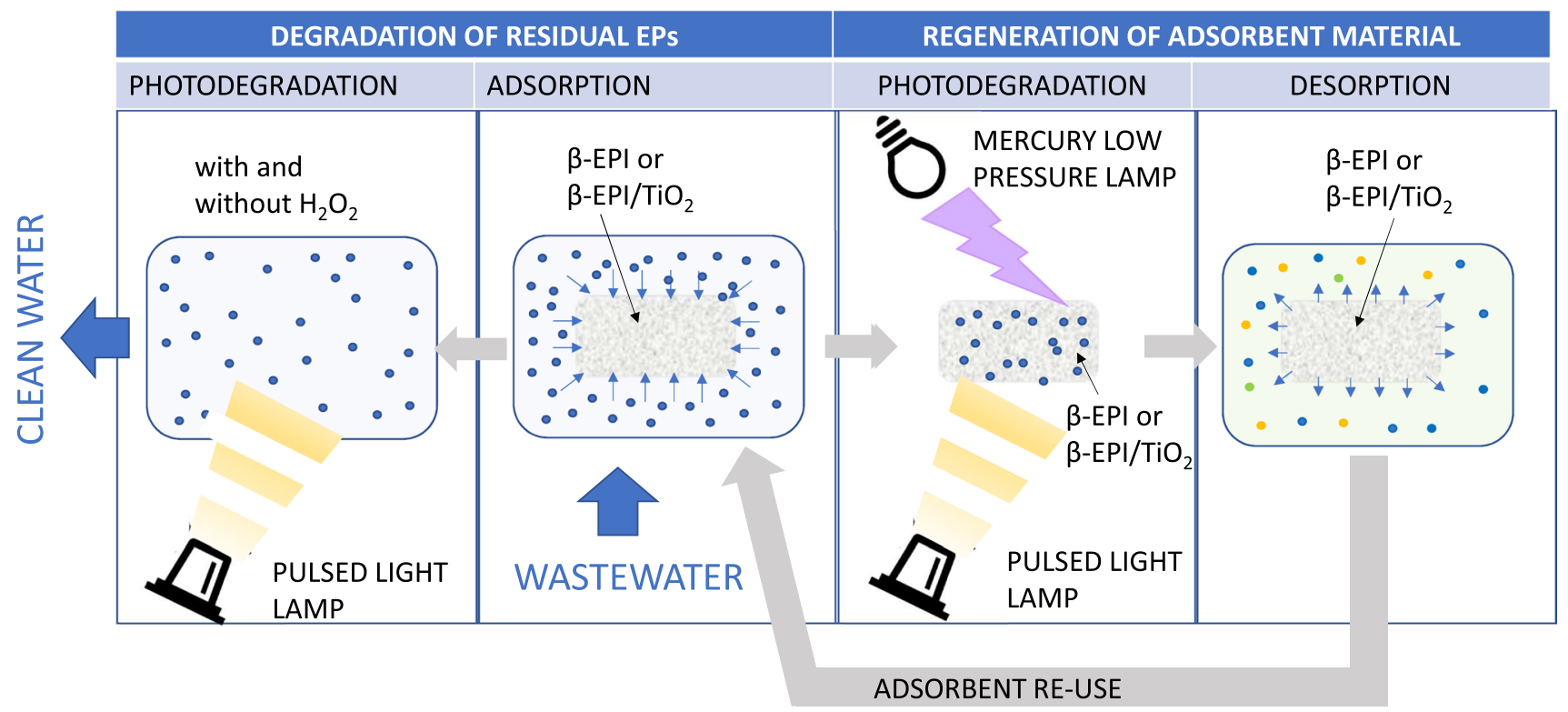

Keywords Adsorbent regeneration $\cdot$ Adsorption $\cdot$ Emerging contaminants $\cdot$ Photocatalytic degradation $\cdot$ Polymers $\cdot$ Pulsed light

\section{Introduction}

The daily use of chemical compounds for domestic, hospital, agricultural and industrial purposes has inevitably led to their release in water (Bunke et al. 2019), creating great concern among the scientific community regarding their impact on the ecosystem and human beings. In particular, great attention is focused on the "emerging pollutants" (EPs), which include pharmaceuticals, personal care products, illicit drugs, pesticides, preservatives, surfactants, plasticizers, flame retardants, etc. (Lopez De Alda et al. 2003; Rodil et al. 2009, 2012; Peña-Guzmán et al. 2019), which are not currently subject to routine environmental monitoring programs nor emission control regime.

These substances might be subject to future legislation due to their adverse effect on the environment and human health (Geissen et al. 2015; Dulio et al. 2018; Bunke et al. 2019). Indeed, EPs present suspected mutagenicity, teratogenicity, and carcinogenicity to humans and other animals (Lei et al. 2015; Bilal et al. 2019), and many of them are recognized as endocrine disruptors (Tobajas et al. 2017; Di Poi et al. 2018).

Sulfamethoxazole ((4-amino- $N$-(5-methyl-1,2-oxazol-3-yl) benzenesulfonamide-SMX) is an antibiotic belonging to the category of sulfonamide drugs, characterized by a methyl substitute in the 5 th position and a 4-aminobenzenesulfonamide group in the fourth position
(Prasannamedha and Kumar 2020). SMX is commonly used to treat digestive infections, typhoid fever, bronchopulmonary and urinary tract infections in humans and animals (Mourid et al. 2019). After consumption, SMX is metabolized to a maximum of $70 \%$ : the remainder, along with its metabolites, is excreted, ending in wastewater (Bizi 2020), and passes unaltered in the environment, where it constitutes a threat to the ecosystem.

Indeed, conventional water treatment techniques, such as biological processes, filtration, coagulation/flocculation, and sedimentation, present limits in EP removal efficiencies (Salazar et al. 2020). A valuable and promising alternative to remove EPs from treated and wastewater is adsorption (Sophia and Lima 2018), thanks to its high efficiency, low cost, and simplicity (Martín et al. 2018). Depending on the material used, adsorption can be very advantageous in terms of biocompatibility and environmental sustainability: several "bio-based" adsorbents, such as chitosan and cyclodextrin polymers, and industrial wastes, such as olive pomace, are very "green", cheap, harmless, and allow effective removal of several categories of EPs. Above all, they can be regenerated and reused multiple times, thus also promoting a circular economy (Rizzi et al. 2017, 2018, 2019, 2020**; Romita et al. 2019).

Cyclodextrin-based polymers are good candidates for the removal of EPs from water (Morin-Crini et al. 
2018). Cyclodextrins are natural cyclic oligosaccharides converted from starch; they present hydrophobic internal cavities able to form inclusion complexes with various low-polarity organic compounds (Murai et al. 1998) and external hydrophilic surfaces which can adsorb polar molecules (Tian et al. 2021). By cross-linking cyclodextrins, it is possible to obtain water-insoluble materials, thus easily separable, to be used for environmental purposes (Morin-Crini et al. 2018). Compared to traditional adsorbents, cyclodextrin-based adsorbents have the advantages of low price, simple synthesis, and high removal efficiency (Tian et al. 2021). Cross-linking of cyclodextrins with epichlorohydrin (1-chloro-2,3-epoxypropane-EPI) is a basic method to produce these adsorbents (Gidwani and Vyas 2014): EPI is a bi-functional agent containing two reactive functional groups, an epoxide group and a chloroalkyl moiety, which can form bonds with $\beta-C D$ molecules (cross-linking step) and/or itself (polymerization step). Despite EPI's toxicity through contact and inhalation, its use is very advantageous since it has a high reactivity and reaction yield. As a cross-linker, EPI provides good mobility to the polymer network and a remarkable hydrophobic character (Gómez-Morte et al. 2021).

Adsorption does not always allow complete and quick removal of EPs from wastewater. Many EPs require long contact times that are incompatible with real applications or are characterized by absorption efficiencies less than $80 \%$. Even in the most favorable case of EPs having high adsorption yields and short contact times, there is still the need to be able to regenerate the adsorbent material. Advanced oxidation processes (AOPs) and pulsed light (PL) can be possible solutions both for the removal of unabsorbed EP remaining in solution and for the regeneration of the adsorbent material.

The photochemical advanced oxidation processes (AOPs) are potentially able to decontaminate wastewater from EPs: the synergistic action of direct photolysis and the reactions with hydroxyl radicals promotes the degradation of a wide range of contaminants (Wols and Hofman-Caris 2012). These radicals can be initiated by photons in the presence of oxidizing agents like hydrogen peroxide and/or a photocatalyst (Rizzi et al. 2015), such as titanium dioxide (Deng and Zhao 2015). For this purpose, low-pressure mercury lamps emitting continuous light centered at $254 \mathrm{~nm}$ are typically used. An interesting alternative to this treatment is the application of pulsed light (PL), i.e., a non-thermal process with promising applications in the food industry, consisting of a successive repetition of short-time, intense and high-power light pulses of broadband emission spectrum including a considerable amount of light in the short-wave UV range (UV-C) (Gómez-López et al. 2007; Baranda et al. 2017). Compared to conventional (continuous) UV light, PL treatment is fast, effective, and more efficient in converting electrical energy into photon energy (Garvey et al. 2015). It delivers powers of the order of $\mathrm{MW}$, without requiring excessive energy consumption (Martínez-López et al. 2019) and uses a broadband light emission which promotes a wide range of chemical compound photodegradation, including those unable to absorb the small wavelength range, centered at $254 \mathrm{~nm}$, emitted by the UV lamp (Gómez-Morte et al. 2021). Recent studies have indeed shown that PL technology can be used to remove pollutants from water, including textile dyes (Serrano-Martínez et al. 2020), pesticides (Baranda et al. 2017), and pharmaceuticals (Gómez-Morte et al. 2021).

In this work, sulfamethoxazole (SMX) removal from water through adsorption onto beta-cyclodextrin-epichlorohydrin copolymer ( $\beta$-EPI) was studied, assessing the influence of adsorbent dosage, initial SMX concentration, $\mathrm{pH}$, and temperature on the process. The obtained information on the effect of experimental parameters on the adsorption was used to promote the desorption of SMX from the polymer and hence to regenerate the adsorbent material. The adsorbed SMX photodegradation on the solid state through continuous irradiation with UV light and PL was attempted as an alternative method to regenerate the polymer alone and in the presence of titanium dioxide as a catalyst. For this purpose, $\beta$-EPI adsorbents bearing different amounts of titanium dioxide were synthesized and tested. Moreover, PL technology was applied as a complementary technique to photodegrade residual SMX in solution after adsorption, also studying the effect of the presence of hydrogen peroxide on the photodegradation process.

This study was carried out between 2019 and 2020 in Bari (Italy) and in Murcia (Spain).

\section{Materials and methods}

\section{Chemicals}

All the chemicals and the reagents used in this work were of analytical grade and were purchased from Sigma Aldrich, except for Aeroxide $\mathrm{TiO}_{2}$ P25 nanoparticles, which were purchased by Evonik Industries AG.

10 and $15 \mathrm{mg} / \mathrm{L}$ sulfamethoxazole stock solutions (SMX-formula: $\mathrm{C}_{10} \mathrm{H}_{11} \mathrm{~N}_{3} \mathrm{O}_{3} \mathrm{~S} ; \mathrm{MW}=253.28 \mathrm{~g} / \mathrm{mol}$ ) were prepared by dissolution of analytical-grade powder (Sigma Aldrich) in deionized water or HPLC-grade water, according to the kind of experiment. The systems at concentrations lower than $10 \mathrm{mg} / \mathrm{L}$ were prepared by dilution of the stock ones. When necessary, the $\mathrm{pH}$ of the SMX solutions was adjusted by using concentrated $\mathrm{HCl}$ and $\mathrm{NaOH}$ solutions. 


\section{Synthesis of $\beta$-cyclodextrin-epichlorohydrin and $\beta$-c yclodextrin-epichlorohydrin- $\mathrm{TiO}_{2}$ polymer}

The $\beta$-cyclodextrin-epichlorohydrin polymer $(\beta$-EPI) was synthesized by following the procedure reported by Pellicer et al. (2019) and modified by Romita et al. (2019). To obtain a final product with 1:25 CD:EPI molar ratio, $24 \mathrm{~g}$ of $\beta$-cyclodextrin and $60 \mathrm{mg}$ of sodium borohydride were dispersed in $24 \mathrm{~mL}$ of deionized water, and the system was left under stirring at $50{ }^{\circ} \mathrm{C}$ for $10 \mathrm{~min}$, then $26 \mathrm{~mL}$ of $40 \%$ (w/v) $\mathrm{NaOH}$ were added, and subsequently, $41.25 \mathrm{~mL}$ of epichlorohydrin (EPI) were introduced dropwise, keeping the system under agitation at $50{ }^{\circ} \mathrm{C}$ for $5 \mathrm{~h}$. The so formed gel was washed four times according to the following order: first with $100 \mathrm{~mL}$ of acetone, then twice with $100 \mathrm{~mL}$ of water, and finally with $100 \mathrm{~mL}$ of acetone. The acetone used for the washing was collected and reused. After the washing procedure, the polymer was dried overnight at $50{ }^{\circ} \mathrm{C}$ in an oven and ground in a mortar to obtain fine particles. Then, it was weighed and suspended in a $10^{-2} \mathrm{M}$ hydrochloric acid solution under stirring ( $25 \mathrm{~g} / \mathrm{L}$ of solution) until neutralization was obtained and rewashed with water $(25 \mathrm{~g} / \mathrm{L}$ of solution). Finally, the product was recovered by decantation, dried overnight at $50{ }^{\circ} \mathrm{C}$, and ground again into fine particles having a size between 100 and 500 microns.

The procedure adopted to prepare $\beta$-EPI polymer bearing titanium dioxide $\mathrm{P} 25$ nanocrystals $\left(\beta\right.$-EPI-TiO $\left.{ }_{2}\right)$ is analogous: the only difference is that the desired amount of $\mathrm{TiO}_{2}$ was added in the $\mathrm{CD}-\mathrm{NaBH}_{4}$-water mixture at the beginning of the synthesis.

\section{UV-Visible Light (UV-Vis) measurements}

For monitoring the SMX concentration in solution during the experiments, UV-Vis spectra of the pollutant were recorded using a Varian CARY 5000 UV-Vis/near-infrared (NIR) spectrophotometer (Varian Inc., now Agilent Technologies Inc.). The wavelength range selected for the recording was 200-500 nm, while the scan rate was set to $1 \mathrm{~nm} / \mathrm{s}$. The maximum adsorption intensity of SMX was observed at $257 \mathrm{~nm}$. For determining the analyte amounts in solution during the tests, external calibration of SMX was performed by preparing and analyzing SMX standard solutions at concentrations ranging from 0.5 to $15 \mathrm{mg} / \mathrm{L}$.

\section{In-batch SMX adsorption and desorption experiments}

For the assessment of the SMX adsorption efficiencies of $\beta$-EPI and $\beta$-EPI-TiO ${ }_{2}$, in-batch experiments were performed by dispersing appropriate amounts of adsorbent in $15 \mathrm{~mL}$ of the pollutant solutions at various concentrations and keeping the system under magnetic stirring at $170 \mathrm{rpm}$ and at temperature costant. At specific contact time values, the systems were centrifuged for $10 \mathrm{~min}$ at $13500 \mathrm{~g}$, and the supernatants were analyzed by UV-Vis spectrophotometry, following the absorption peak at $257 \mathrm{~nm}$ to infer SMX residual concentrations. The adsorption efficiencies were expressed as a percentage of SMX retained onto the polymer, calculated by using the following equation (Eq. 1):

$\operatorname{SMX}$ adsorption $(\%)=\frac{A_{0}-A_{t}}{A_{0}} \times 100$

where $A_{0}$ and $A_{t}$, respectively, are the SMX absorbance at time zero and at the time of the analysis.

The influence of contact time, polymer amount, SMX initial concentration, and temperature on SMX adsorption onto $\beta$-EPI were evaluated by varying one parameter at a time and fixing the other ones.

For evaluating the effect of $\mathrm{pH}$ on SMX adsorption onto $\beta$-EPI, in-batch experiments were performed at $\mathrm{pH}$ values 2.0, 4.5, 7.0, 9.5, and 12.0, obtained by using appropriate amounts of $\mathrm{HCl}$ and $\mathrm{NaOH}$. For each test, a solution containing $10 \mathrm{mg} / \mathrm{L} \mathrm{SMX}$ and $25 \mathrm{mg} / \mathrm{mL}$ of the polymer was used.

The possibility of recovering and reusing $\beta$-EPI and $\beta$-EPI-TiO ${ }_{2}$ was also investigated, by conducting in-batch desorption experiments in different extraction media, such as organic solvents and water, at specific conditions. The polymer bearing pre-adsorbed SMX were dispersed in $15 \mathrm{~mL}$ of solvent under magnetic stirring at $170 \mathrm{rpm}$; the aliquots of the batch were withdrawn at specific times, then centrifuged at $13500 \mathrm{~g}$ for $10 \mathrm{~min}$ and the concentrations of SMX released were determined at the UV-Vis spectrophotometer $(\lambda=257 \mathrm{~nm})$. The desorption efficiencies were calculated employing the following equation (Eq. 2):

$\operatorname{SMX}$ desorption $(\%)=\frac{m_{\mathrm{des}}}{m_{\mathrm{ads}}} \times 100$

where $m_{\mathrm{des}}$ and $m_{\mathrm{ads}}$ are the amount of SMX desorbed at the time of the analysis and the amount of pollutant adsorbed before the release experiment, respectively.

\section{Differential scanning calorimetry (DSC)}

The thermal properties of the $\beta$-EPI polymer after different cycles of adsorption/desorption were investigated by DSC (Q200 TA Instruments) under a nitrogen flow of $50 \mathrm{~mL} / \mathrm{min}$ at the heationg rate of $10{ }^{\circ} \mathrm{C} / \mathrm{min}$ from 0 to $400{ }^{\circ} \mathrm{C}$.

\section{Photodegradation of SMX via a UV lamp}

The photodegradation of SMX on the solid state was performed by dispersing an appropriate amount of $\beta$-EPI or 
$\beta$-EPI-TiO ${ }_{2}$ bearing SMX pre-adsorbed in water, at $25 \mathrm{mg} /$ $\mathrm{mL}$ fixed ratio, and irradiating the system with a UV lamp having a continuous source with maximum emission at $\lambda=254 \mathrm{~nm}$ (Spectroline, Model CNF 280C/FE, light flow $0.2 \mathrm{~mW} / \mathrm{cm}^{2}$ ).

For estimating SMX photodegradation's efficiency, the release of the pollutant from the polymer was carried out right after the UV light treatment. Assuming that the whole amount not released is photodegraded, i.e., that there are no intact SMX molecules still bound to the adsorbent, the percentage of SMX photodegraded was calculated by using the following equation (Eq. 3):

SMX photodegradation $(\%)=\frac{m_{\text {ads }}-m_{\text {des }}}{m_{\text {ads }}} \times 100$

The amounts of SMX adsorbed, photodegraded, and released were monitored by using UV-Vis spectroscopy $(\lambda=257 \mathrm{~nm})$.

\section{Photodegradation of free or adsorbed SMX via pulsed light $(P L)$}

The photolysis of SMX in an aqueous solution through pulsed light (PL) was performed, and the effect of the presence of $1 \mathrm{mM}$ hydrogen peroxide on the process was evaluated. Also, $\beta$-EPI or $\beta$-EPI-TiO 2 suspensions after SMX adsorption were subjected to PL and subsequently to release experiments in the water at $80{ }^{\circ} \mathrm{C}$ to evaluate the efficiency of photodegradation on solid-state.

For all the PL tests, $20 \mathrm{~mL}$ of $10 \mathrm{mg} / \mathrm{L}$ aqueous solutions, or $20 \mathrm{~mL}$ of $25 \mathrm{mg} / \mathrm{mL} \beta$-EPI or $\beta$-EPI-TiO ${ }_{2}$ suspension, were placed in a Petri dish without cover, in a Steribeam Xe-MaticA-Basic-1 L PL system. A discharge voltage of $2.5 \mathrm{kV}$ was chosen, producing light with an energy of $500 \mathrm{~J} /$ pulse (2.5 MW) and $21 \%$ UV component. The fluence incident on the sample surface was $2.14 \mathrm{~J} / \mathrm{cm}^{2}$ per pulse. Different fluences were provided to samples by treating them with multiple pulses. UV-Vis spectroscopy did not allow an accurate estimation of the residual concentration of SMX in solution at different times since the spectra of both SMX and photodegradation by-products resulted in being overlapped. Therefore, the quantification of SMX was carried out via high-performance liquid chromatography (HPLC) by using an Agilent 1200 HPLC instrument with a photodiode-array UV-Vis detector and a Zorbax Eclipse Plus analytical column, $\mathrm{C}_{18}$ reverse-phase type $(4.6 \times 100 \mathrm{~mm}, 3.5 \mu \mathrm{m})$ (Trovó et al. 2009; Długosz et al. 2015). For each run, $5 \mu \mathrm{L}$ of a sample, properly treated with a Chromafil Xtra PA-45/25 $0.45 \mu \mathrm{m}$ filter, were injected, and an isocratic elution was accomplished at $0.8 \mathrm{~mL} / \mathrm{min}$ and $30^{\circ} \mathrm{C}$ with a mobile phase composed of $70 \%$ of formic acid $(0.1 \% \mathrm{v} / \mathrm{v})$ and $30 \%$ of acetonitrile; the wavelength selected to determine SMX was
$270 \mathrm{~nm}$. Under the operating conditions selected, the SMX retention time was $3.5 \mathrm{~min}$, and its quantification limit was less than $0.5 \mathrm{mg} / \mathrm{L}$. The instrument's external calibration was made by preparing and analyzing SMX standard solutions at concentrations ranging from 0.5 to $10 \mathrm{mg} / \mathrm{L}$. When samples collected from PL experiments at different times were analyzed, two other peaks appeared in the chromatograms at 1.1 and 1.6 min with maximum light absorption at 249 and $267 \mathrm{~nm}$, respectively. To qualitatively assess the possible degradation of these by-products via PL, further runs were repeated by setting the reading wavelength at 249 and $267 \mathrm{~nm}$.

All experiments were replicated five times. The statistical analysis of replicates was used to evaluate the relative standard deviation.

\section{Results and discussion}

\section{Effect of contact time $\beta$-EPI dosage, SMX initial concentration, $\mathrm{pH}$ and temperature on SMX adsorption}

The adsorption process of SMX on $\beta$-EPI polymer was studied as a function of some experimental parameters which determine the characteristics that the laboratory prototype must have, in order to obtain a good SMX removal from water. Conditio sine qua non to scale up the process is to have a good adsorption capacity in a short time. If the contact time between the adsorbent/wastewater necessary to reach the adsorption equilibrium is too long, it is not possible to work in flux and the field of possible applications in real-life situations is greatly narrowed. The effects of contact time and adsorbent dosage on SMX adsorption onto $\beta$-EPI are shown in Fig. 1a. When $3.125 \mathrm{mg} / \mathrm{mL}$ of $\beta$-EPI were used, the equilibrium was reached right after $5 \mathrm{~min}$, while the plateau was reached within 10-15 min in the presence of higher doses of material. This result is relevant since most of the materials tested as adsorbents for SMX, such as biochars, carbon nanotubes, many different activated carbons, graphene and its derivatives, polymeric substances, and minerals require long contact times (Wang et al. 2020; Zheng et al. 2020; Prasannamedha and Kumar 2020). For example, coal-based activated carbons require a contact time of $5 \mathrm{~h}$ to remove $55 \%$ of SMX (Çalışkan and Göktürk 2010), and F400 granular activated carbon requires up to $90 \mathrm{~h}$ with a removal of $80 \%$ (Moral-Rodríguez et al. 2016).

Moreover, by incrementing the amount of $\beta$-EPI in the batch, the SMX adsorption percentages increase: by switching from 3.125 to $25 \mathrm{mg} / \mathrm{mL}$ polymer dosage, the maximum SMX uptake changes from 25 to $61 \%$. This is a very common dose-effect and can be attributed to the greater 
Fig. 1 SMX adsorption efficiencies onto $\beta$-EPI polymer at different contact times and adsorbent dosages: $3.125 \mathrm{mg}$ / $\mathrm{mL}$ (black), $6.25 \mathrm{mg} / \mathrm{mL}$ (red), $12.5 \mathrm{mg} / \mathrm{mL}$ (blue) and $25 \mathrm{mg} /$ $\mathrm{mL}$ (green) (initial SMX concentration: $10 \mathrm{mg} / \mathrm{L})(\mathbf{a})$; SMX adsorption efficiencies onto $\beta$-EPI polymer at different pollutant concentration using $25 \mathrm{mg} / \mathrm{L}$ of $\beta$-EPI $25 \mathrm{mg} / \mathrm{mL}$ and a contact time of $15 \mathrm{~min}$ (b)

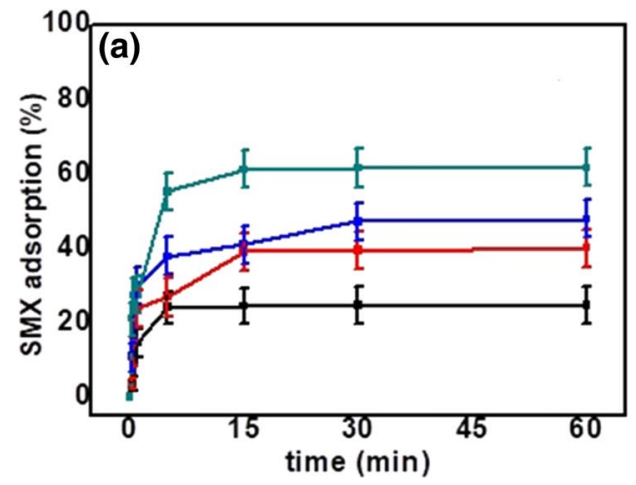

availability of the active sites or surface area to the pollutant molecules at higher adsorbent doses (Çalışkan and Göktürk 2010; Romita et al. 2019).

Regarding the influence of SMX initial concentration on the process, it was observed that the efficiency of SMX adsorption increased from 25 to $69 \%$, at increasing of the initial concentration of the pollutant (Fig. 1b) as already observed using the same polymer to remove the azo dye Direct Red 83:1 from water (Pellicer et al. 2019). However, in the case of atrazine, at increasing the initial pollutant concentration, a slight decrease of the removal efficiency was observed (Romita et al. 2019). The increase of the adsorption at the increase of the SMX concentration suggests that molecular diffusion has an important role in the adsorption process.

Figure 2 a shows that $\mathrm{pH}$ remarkably affects SMX adsorption. This effect is mainly due to the protonation or deprotonation of SMX. Indeed, the adsorbent does not present or acquire any charge in the $\mathrm{pH}$ range 2-12 as already observed in previous studies (Romita et al. 2019).

The SMX has two $p K_{a}$ values: $p K_{a 1}=1.6$, corresponding to the aniline N's protonation, and $p K_{a 2}=5.7$, relative to the sulfonamide deprotonation. The maximum adsorption efficiency, 84 and $87 \%$, respectively, were obtained at $\mathrm{pH} 2.0$ and 4.5, which are between $p K_{a 1}$ and $p K_{a 2}$ (Rezende et al. 2019) where SMX exists mostly as $\mathrm{SMX}^{0}$. This result suggests that weak hydrophobic interactions are more important in the adsorption process onto $\beta$-EPI, than electrostatic ones, contrary to that reported in the literature in the case of SMX adsorption on activated carbons (Prasannamedha and Kumar 2020). From pH 7.0 to 12.0 , i.e., when $\mathrm{SMX}^{-}$anions concentration increased, the adsorption percentages decreased dramatically, to only $9 \%$ in the case of pH 12.0 (Fig. 2a). A similar trend was observed in the study of the $\mathrm{pH}$ effect on bisphenol $\mathrm{A}$ adsorption onto $\beta$-EPI (Kitaoka and Hayashi 2002). The presence of interactions mainly hydrophobic between SMX and $\beta$-EPI polymer is in accordance with the complex structure of the polymer, which allows for the formation of inclusion and association complexes and the hydrophobic character of the SMX (Romita et al. 2021).

At increasing of the temperature from 277 to $333 \mathrm{~K}$ (Fig. 2b), SMX adsorption (10 mg/L SMX/25 mg/L $\beta$-EPI polymer) decreases from 71 to $27.0 \%$, as already observed studying the adsorption of atrazine on the same material. This trend indicates that the adsorption process is exothermic. It is advantageous because it permits the reduction of energy consumption in adsorption and suggests the possibility of using hot water to promote SMX desorption regenerating the adsorbent material.

\section{SMX desorption and $\beta$-EPI reuse}

In order to promote a circular economy, the possibility of recovering and reusing the adsorbent was also assessed. Generally, cyclodextrin-epichlorohydrin polymers postsorption treatment with organic solvents, such as alcohols,
Fig. 2 Efficiencies of $10 \mathrm{mg} / \mathrm{L}$ SMX adsorption onto $\beta$-EPI polymer $(25 \mathrm{mg} / \mathrm{mL})$ at different $\mathrm{pH}$ values (a) and temperatures (b) (contact time: $15 \mathrm{~min}$ )
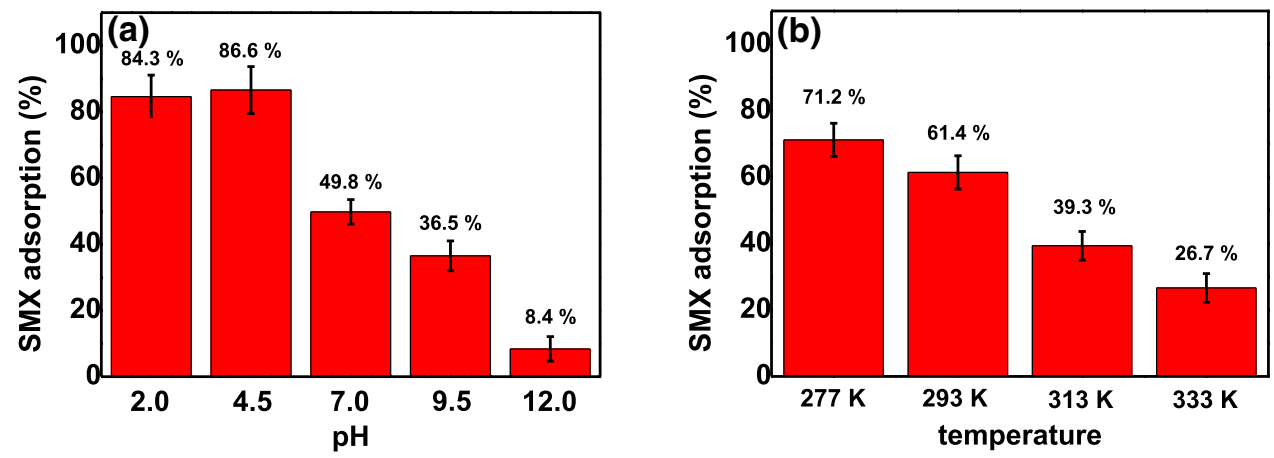
is very effective for releasing organic pollutants (Orprecio and Evans 2003; Morin-Crini et al. 2018).

Since SMX is more soluble in water than other categories of organic pollutants, also considering that it is present mostly in its anionic form at neutral $\mathrm{pH}$, it was hypothesized that there might be alternative methods for its desorption from $\beta$-EPI, as for example, the use of operating conditions which inhibit the adsorption process, such as basic environment or high temperatures. On this grounds, was not only ethanol tested as SMX release medium, but also water at 293, 333, $353 \mathrm{~K}$ and water at $\mathrm{pH} 12.0$. Before each experiment, a preliminary 15 min-long adsorption of $10 \mathrm{mg} / \mathrm{L}$ SMX was performed by using $25 \mathrm{mg} / \mathrm{mL}$ of $\beta$-EPI, followed by a polymer washing step with deionized water to remove any traces of exhausted SMX solution. Results are reported in Fig. 3a. It was observed that water at $293 \mathrm{~K}$ allowed a maximum SMX extraction of only $32 \%$ after $15 \mathrm{~min}$ of
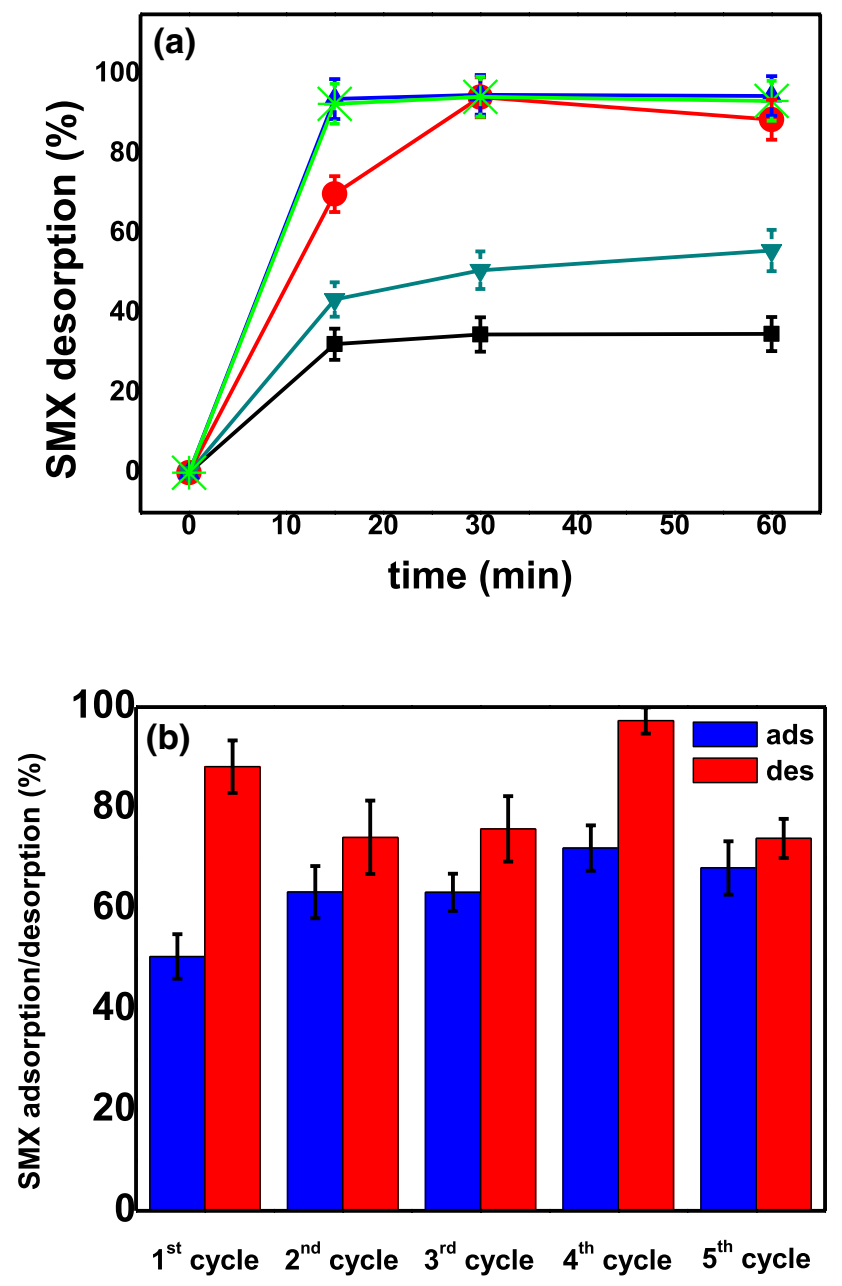

Fig. 3 Efficiencies of SMX desorption from $\beta$-EPI $(25 \mathrm{mg} / \mathrm{mL})$ under different conditions: water at $293 \mathrm{~K}(\boldsymbol{\square})$, water at $333 \mathrm{~K}(\boldsymbol{\bullet})$, water at $353 \mathrm{~K}(\boldsymbol{\Delta})$, etanol ( $\boldsymbol{\nabla})$, water at $\mathrm{pH} 12$ (X) (a). Efficiencies of multiple SMX $(10 \mathrm{mg} / \mathrm{L})$ adsorption and desorption cycles performed with the same $\beta$-EPI amount $(25 \mathrm{mg} / \mathrm{mL})(\mathbf{b})$ contact, then the plateau was reached. The use of ethanol allowed the release of $56 \%$ of the pollutant within $60 \mathrm{~min}$. As expected, by working at $\mathrm{pH} 12$, a high SMX desorption efficiency was obtained: $93 \%$ of SMX was recovered from $\beta$-EPI within only $15 \mathrm{~min}$. Very interesting results were found by using hot water for SMX extraction from the adsorbent: at $333 \mathrm{~K}, 94 \%$ of desorption efficiency was reached within $30 \mathrm{~min}$, then it slightly decreased at $60 \mathrm{~min}$. At $353 \mathrm{~K}$ the process was faster, with a maximum SMX release of $94 \%$ after only $15 \mathrm{~min}$. Although analogous findings were obtained working at room temperature at $\mathrm{pH} 12.0$, it should be considered that several consecutive $\beta$-EPI washing steps would be needed in order to neutralize the polymer and remove any traces of $\mathrm{OH}^{-}$anions adsorbed in order not to lose the good SMX adsorption properties of the polymer. This would require the use of large amounts of water and an increase in the duration of the processes. On the contrary, the use of hot water for SMX release from $\beta$-EPI would be advantageous, thanks to its high rapidity and feasibility but disadvantageous for the energetic cost associated with the heating of water. Despite this limitation, $\beta$-EPI regeneration in water at $353 \mathrm{~K}$ was chosen for the subsequent experiments, and the possibility of reusing the adsorbent multiple times was investigated. A similar approach based on the use of water at high temperatures was also used to promote the desorption of textile dyes from chitosan films (Rizzi et al. 2018).

Five consecutive cycles of SMX adsorption and desorption were performed by using $25 \mathrm{mg} / \mathrm{mL}$ of polymer, $10 \mathrm{mg} / \mathrm{L}$ SMX solution, and water at $353 \mathrm{~K}$. The batch volume was the same for all the cycles $(15 \mathrm{~mL})$ and the duration of each cycle was set to $15 \mathrm{~min}$, with an intermediate polymer washing step.

As shown in Fig. 3b, high SMX adsorption and desorption efficiencies were found, and the system was still efficient also in the subsequent cycles, despite the use of a high temperature for the pollutant release. Interestingly, the percentage of SMX uptake resulted as enhanced from 50 to over $70 \%$ starting from the second cycle.

An analogous increase in adsorption efficiency in consecutive adsorption/desorption cycles, although of only $10 \%$, was obtained by regenerating the same polymer by using a NaBr 0.5 M (Romita et al. 2021). This could be due to a partial reorganization of the polymer, which would make the polymeric network more accessible to the pollutant (MorinCrini et al. 2018). This finding is confirmed by the shift at a higher and lower temperature of the endothermic peaks associated with the water loss and with the fusion and beginning of the decomposition processes, respectively, observed comparing DSC thermograms of the polymer before the adsorption and after the first and the fifth adsorption/desorption cycle (Fig. 4). Differences were evidenced only by thermal analysis and not by other techniques (SEM and FT-IR, data 


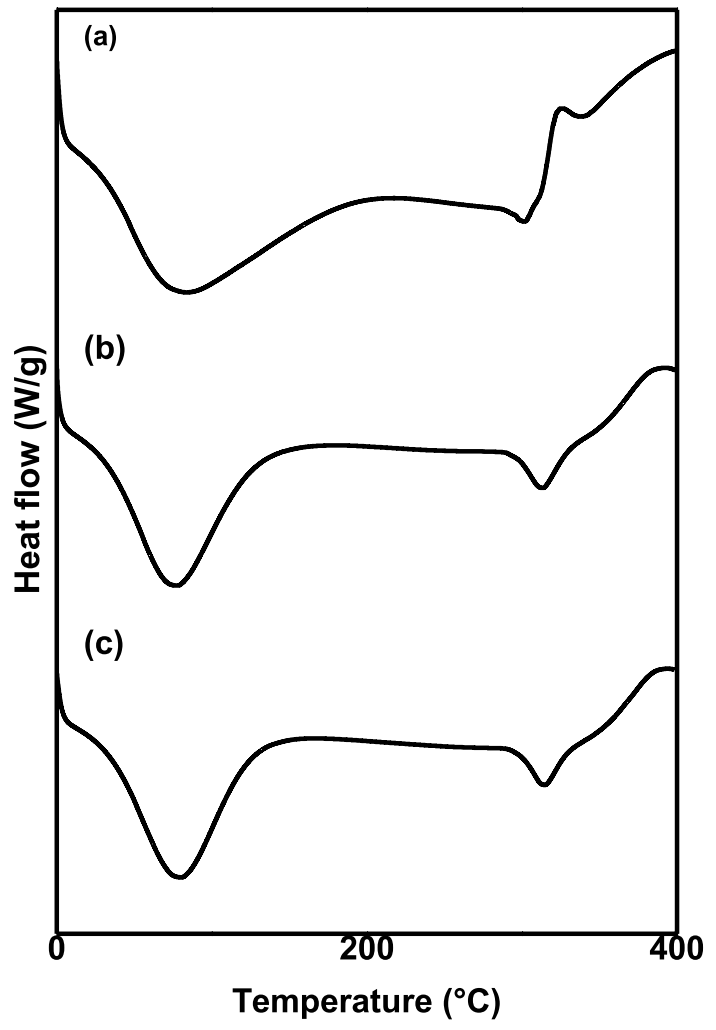

Fig. 4 Comparison of thermograms of $\beta$-EPI polymer swelled before adsorption (a), after the 1th (b) and the 5th (c) adsorption/desorption cycle

not shown) as already observed with another cyclodextrin based polymer which was characterized before and after the regeneration (Rizzi et al. 2021).

Based on these results, it can be asserted that $\beta$-EPI can be reused for SMX removal multiple times, without any loss in performance, making it an efficient, low-cost, and environmentally-friendly material.

\section{$\beta$-EPI-TiO ${ }_{2}$ nanocomposite: effect of photocatalyst dosage on adsorption and UV photodegradation of SMX adsorbed}

As an alternative to desorption, adsorbent material could be regenerated by using advanced oxidation processes (AOP) for the photolysis of adsorbed SMX ( Alharbi et al. 2017; Lam and Mabury 2005; Ryan et al. 2011). This could be done using a polymeric nanocomposite consisting of a cyclodextrin-epichlorohydrin polymer bearing $\mathrm{TiO}_{2}$ nanocrystals in its structure $\left(\beta\right.$-EPI-TiO $\left.{ }_{2}\right)$ for the adsorption and the subsequent photodegradation of the pollutant in a solid-state,

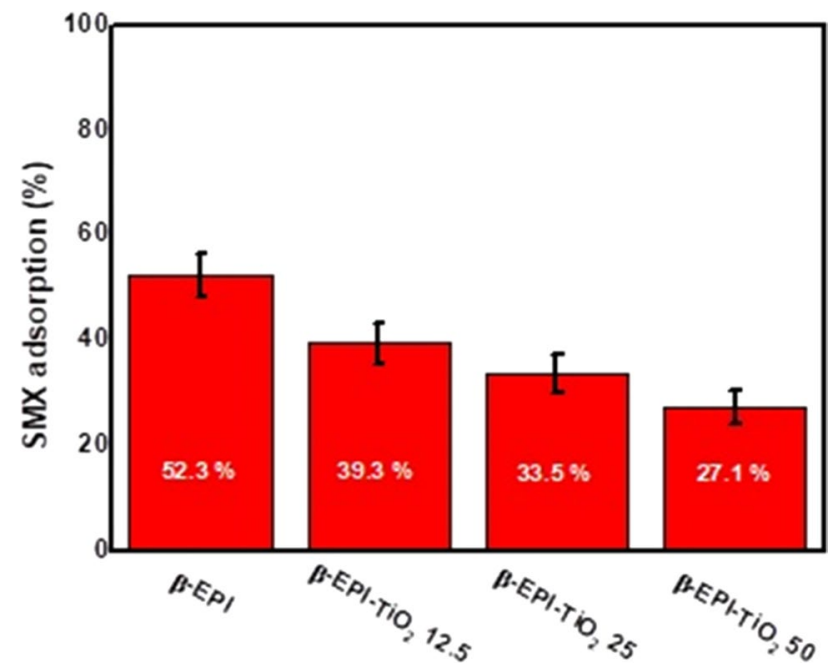

Fig. 5 Efficiencies of $10 \mathrm{mg} / \mathrm{L}$ SMX adsorption onto $\beta$-EPI and $\beta$-EPI-TiO ${ }_{2}$ polymers $(25 \mathrm{mg} / \mathrm{mL})$ with different amounts of photocatalyst, at room temperature and neutral $\mathrm{pH}$ (contact time: $60 \mathrm{~min}$ )

i.e., by directly irradiating the polymer, exploiting the photocatalytic properties of the semiconductor. An analogous approach was carried out with chitosan-based adsorbent materials, with successful results regarding diclofenac and tetracycline removal (Rizzi et al. 2019, 2020).

For this reason, $\beta$-EPI- $\mathrm{TiO}_{2}$ polymers were synthesized by adding $12.5,25$, and $50 \mathrm{mg}$ of titanium dioxide per gram of cyclodextrin used in the reaction mixture (named $\beta$-EPI-TiO $12.5,25$, and 50 , respectively). The adsorption ability of these nanocomposites was assessed, evaluating the influence of photocatalyst dosage on the process. As shown in Fig. 5, the SMX uptake at the equilibrium (i.e., after $60 \mathrm{~min}$ of contact) decreased progressively by incrementing the amount of $\mathrm{TiO}_{2}$ in the polymer structure: it varied from $52 \%$ in the absence of photocatalyst to $39 \%$ in the presence of $50 \mathrm{mg} / \mathrm{g}$ of $\mathrm{TiO}_{2}$. This is due to the presence of charge on the surface of titanium dioxide nanoparticles characterized by a point of zero charge value (PZC) of 5.2 (Min 2013). In other words, at $\mathrm{pH}$ above 5.2, the catalyst surface is negatively charged and repulses the anionic compounds in water (Chong et al. 2010; Min 2013). Therefore, by working at neutral $\mathrm{pH}$, the negative surface charge of the $\mathrm{TiO}_{2}$ nanoparticles may exert repulsive forces towards SMX, which is partly present in its anionic form (Chong et al. 2010): the more $\mathrm{TiO}_{2}$ contained in the polymer, the more hindered SMX adsorption is.

Results of the photodegradation of SMX adsorbed onto the $\beta$-EPI-TiO 2 polymer are reported in Table 1 and are compared with data obtained from control experiments 
Table 1 Comparison of efficiencies of SMX desorption from $\beta$-EPI and $\beta$-EPI-TiO $2(25 \mathrm{mg} /$ $\mathrm{mL}$ ) at different amounts of photocatalyst with and without post-sorption continuous UV photodegradation $(254 \mathrm{~nm})$ of the pollutant on solid state ("dark" versus "UV"); efficiencies of SMX photodegradation on solid state are also compared

\begin{tabular}{lllll}
\hline & $\begin{array}{l}\text { Intermediate step between } \\
\text { adsorption and desorption }\end{array}$ & $\begin{array}{l}\text { SMX adsorp- } \\
\text { tion }(\%)\end{array}$ & $\begin{array}{l}\text { SMX desorp- } \\
\text { tion }(\%)\end{array}$ & $\begin{array}{l}\text { Photodegra- } \\
\text { dation efficiency } \\
(\%)\end{array}$ \\
\hline$\beta$-EPI $\left(\mathrm{NO} \mathrm{TiO}_{2}\right)$ & Dark & 52.3 & 95.8 & - \\
& $\mathrm{UV}$ & 47.0 & 23.2 & 76.8 \\
$\beta$-EPI- $\mathrm{TiO}_{2} 12.5$ & Dark & 39.3 & 94.8 & - \\
& $\mathrm{UV}$ & 41.4 & 18.6 & 81.4 \\
$\beta$-EPI- $\mathrm{TiO}_{2} 25$ & Dark & 33.5 & 94.7 & - \\
$\beta$ & $\mathrm{UV}$ & 41.0 & 26.3 & 73.7 \\
& Dark & 30.8 & 93.6 & - \\
& $\mathrm{UV}$ & 26.9 & 37.8 & 62.2 \\
\hline
\end{tabular}

consisting of adsorption-desorption without intermediate UV irradiation ("dark" step) in order to assess the efficiency of SMX photodegradation. When photodegradation was not performed, more than $90 \%$ of SMX adsorbed was desorbed from all the polymers, confirming the high performance of the high-temperature method for the pollutant release, also in the presence of photocatalyst in the adsorbent. When $\beta$-EPI was irradiated, the subsequent SMX desorption was reduced to $23 \%$, which means that in-situ photodegradation occurred successfully, removing $78 \%$ of the pollutant adsorbed. When using $\beta$-EPI-TiO 2 12.5, SMX photodegradation efficiency slightly increased to $81 \%$, showing a very modest photocatalytic activity of the system. This might be due to the very high photoreactivity of adsorbed SMX under UV light, regardless of the presence of $\mathrm{TiO}_{2}$. By incrementing the amount of photocatalyst, the efficiency of SMX degradation decreased, becoming worse than in the absence of $\mathrm{TiO}_{2}: \beta$-EPI-TiO 25 allowed the destruction of $74 \%$ of SMX, while $\beta$-EPI-TiO 250 provided $62 \%$ of pollutant photodegradation. This could be explained by considering that excessive $\mathrm{TiO}_{2}$ particles in the polymer structure may lead not only to the formation of particle stacks, which would reduce the photocatalysis surface area (Lin and Lee 2010) but also on an enhancement of the light reflectance (Hasmath Farzana and Meenakshi 2013), which would inhibit the photodegradation of SMX adsorbed.

\section{Use of PL for degradation of SMX adsorbed onto the polymer and effect of $\mathrm{TiO}_{2}$}

Figure 6 shows the results of the experiments on the degradation of $10 \mathrm{mg} / \mathrm{L}$ SMX adsorbed onto $25 \mathrm{mg} / \mathrm{mL} \beta$-EPI and $\beta$-EPI-TiO 2 via PL, monitoring the pollutant concentration through HPLC.

The fading of the SMX HPLC signal, due to its photolysis, was accompanied by the appearance of a new signal with a shorter retention time corresponding to a degradation product (by-product 1), which has been identified as sulfanilic acid according to the NIST database (https://webbo ok.nist.gov/cgi/cbook.cgi?ID=C121573\&Mask=400\#UVVis-Spec). This implies the rupture of the $\mathrm{S}-\mathrm{N}$ bond of the SMX molecule during PL treatment. This SMX degradation product with peak absorption at $249 \mathrm{~nm}$ has been detected before in photolysis studies using other light sources (Trovó et al. 2009; Yang et al. 2017; Martínez-Costa et al. 2018). Another SMX degradation product with two absorption peaks at 242 and $267 \mathrm{~nm}$ was detected (by-product 2).

Although the polymers had been washed after SMX adsorption, part of the pollutant was released in water before applying PL. Data reported in Fig. 6a indicate that, in the absence of $\mathrm{TiO}_{2}$, PL promoted the complete degradation of SMX, with the concomitant formation of sulfanilic acid (byproduct 1), but by-product 2 was not observed. As concerns SMX degradation on solid-state, when the treatment was extended to $128 \mathrm{~J} / \mathrm{cm}^{2}$, no by-products were detected, which points toward extensive mineralization. This is confirmed by the absence of both SMX and by-products in the desorption performed on the same polymer sample after PL (Fig. 6b). It is probable that by-product 1 encapsulated inside the polymer is more subject to further photolysis than when free in solution. The inclusion of $\mathrm{TiO}_{2}$ in the polymer at $12.5 \mathrm{mg} / \mathrm{g}$ CD (Fig. 6c) and $50 \mathrm{mg} / \mathrm{g} \mathrm{CD} \mathrm{(Fig.} \mathrm{6e)} \mathrm{also} \mathrm{allows} \mathrm{complete}$ SMX degradation. However, these require higher fluences. It is possible that the $\mathrm{TiO}_{2}$ interferes with the photolysis of SMX by competing with photons rather than generating hydroxyl radicals that would have eventually oxidize the pollutant. The post-PL desorption from the $\beta$-EPI-TiO 212.5 and 50 showed the release of by-product 1 (Fig. $6 \mathrm{~d}$ and $\mathrm{f}$ ), indicating that degradation of sulfanilic acid was not accomplished as may happen in the case of $\beta$-EPI without $\mathrm{TiO}_{2}$. The lower amount of SMX adsorbed onto $\beta$-EPI-TiO 250 , along with the $\mathrm{TiO}_{2}$ stacking, causes a further decrease in 

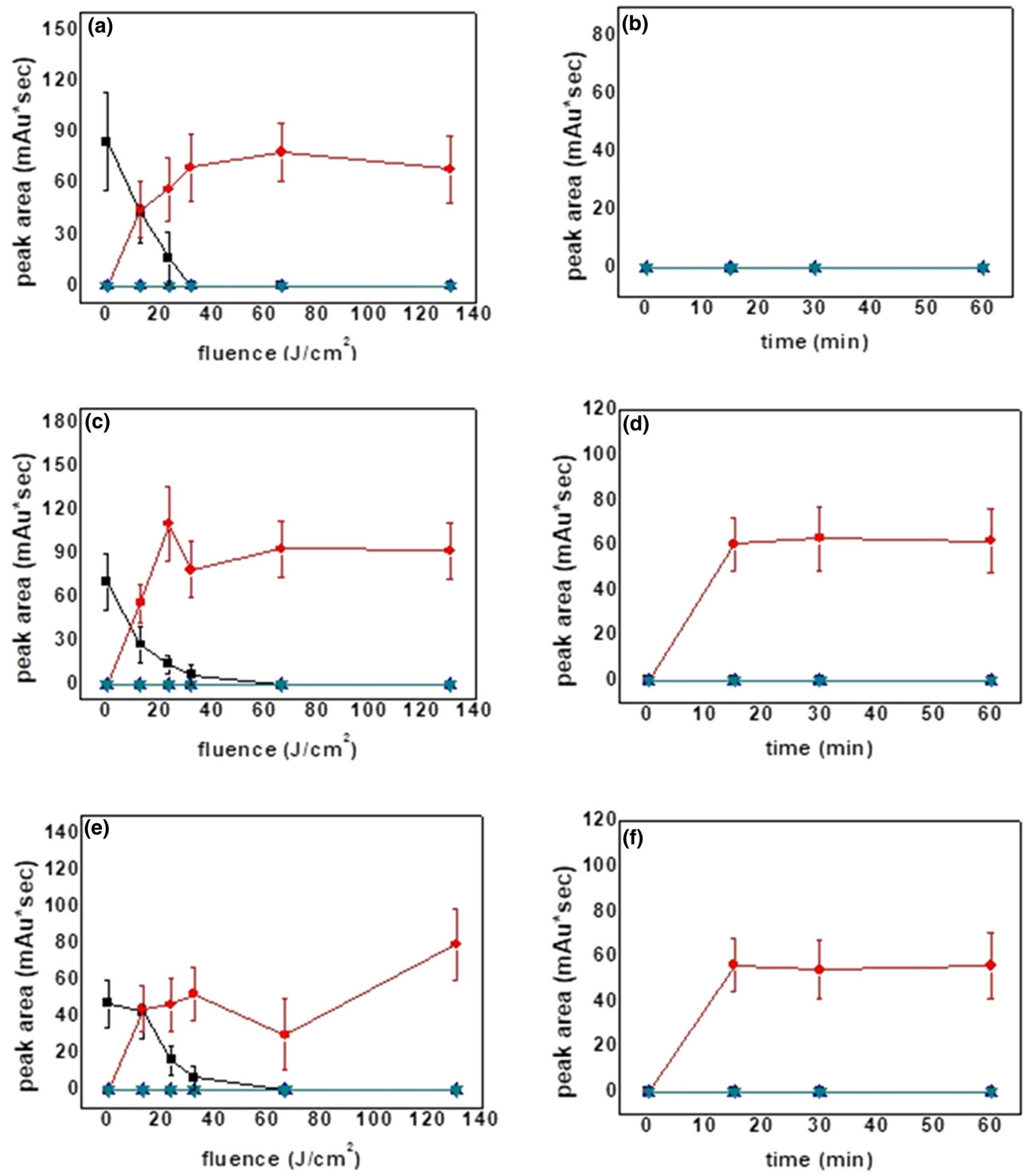

Fig. 6 Degradation of adsorbed SMX on $\beta$-EPI $(25 \mathrm{mg} / \mathrm{mL})$ by PL without (a) and with 12.5 (c) and $50 \mathrm{mg}$ of $\mathrm{TiO}_{2}$ per gram of cyclodextrin (e); post-PL desorption of SMX and by-products from $\beta$-EPI without (b) and with 12.5 (d) and $1.250 \mathrm{mg} \mathrm{TiO}$ per gram of cyclo-

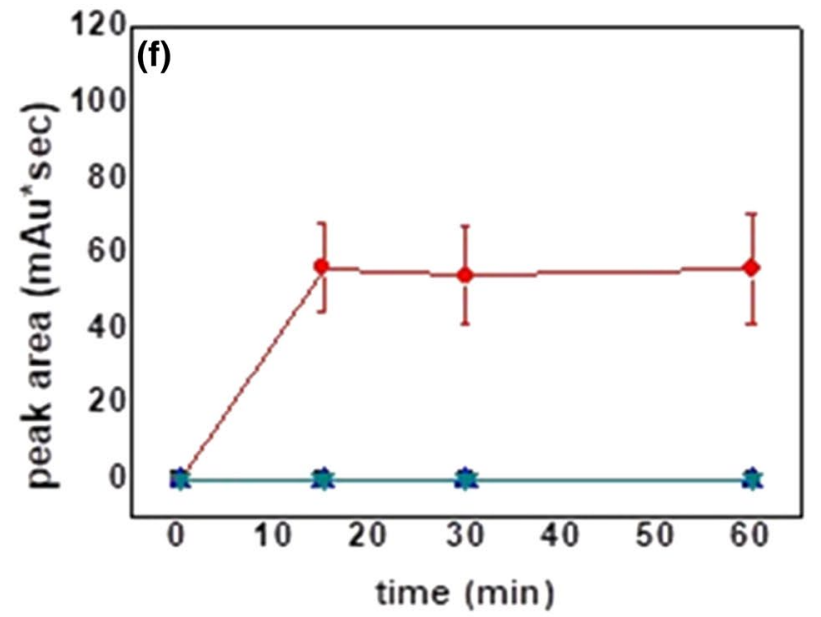

dextrin (f). (ם) indicates SMX, detected at $270 \mathrm{~nm}$; () indicates the by-product 1 , detected at $249 \mathrm{~nm} ;(\boldsymbol{\Lambda})$ indicates the by-product 2 , detected at $267 \mathrm{~nm}$ and $(\boldsymbol{\nabla})$ indicates the by-product 2, detected at $242 \mathrm{~nm}$ 


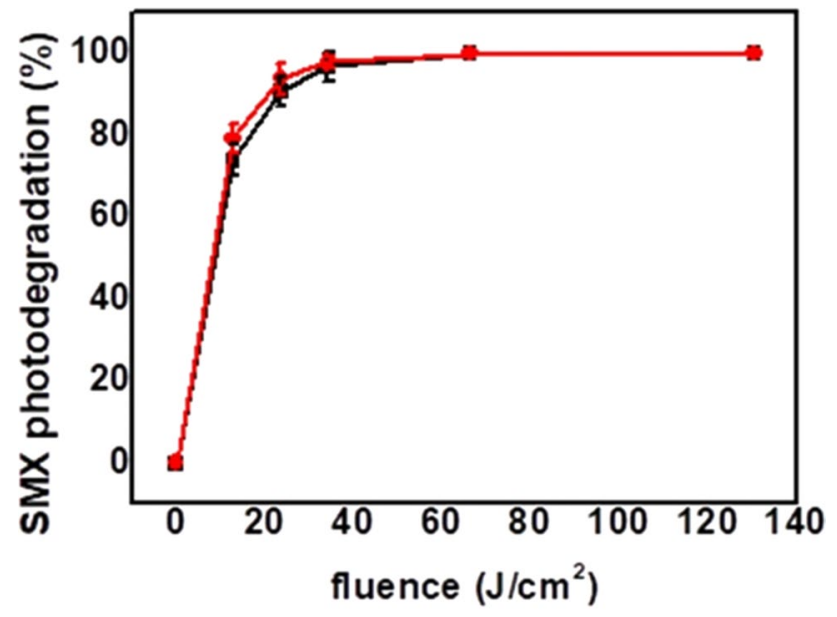

Fig. 7 Comparison between degradation of $10 \mathrm{mg} / \mathrm{L}$ SMX by PL in the absence (ם) and in the presence $(\mathbf{0})$ of $10^{-3} \mathrm{M} \mathrm{H}_{2} \mathrm{O}_{2}$
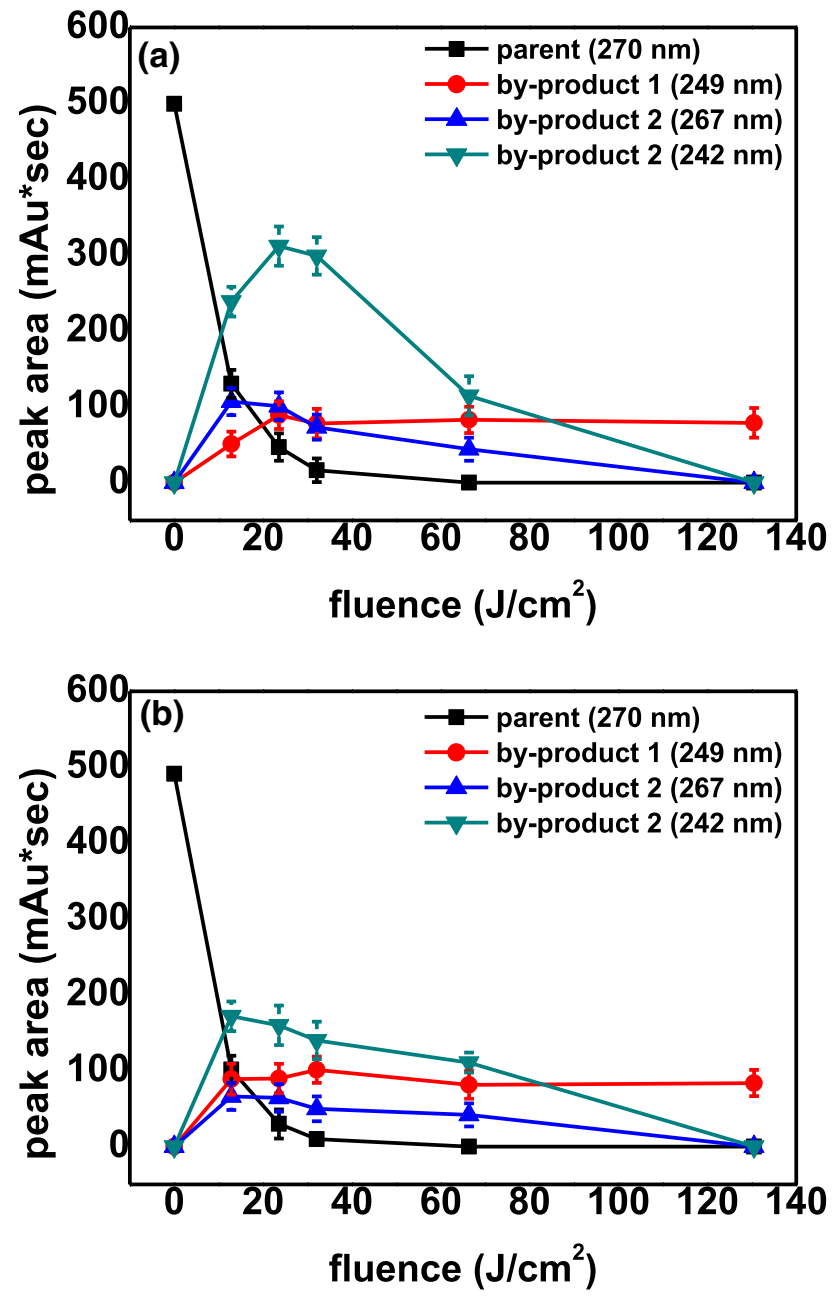

Fig. 8 Degradation of $10 \mathrm{mg} / \mathrm{L}$ SMX by PL without (a) and with (b) $10^{-3} \mathrm{M} \mathrm{H}_{2} \mathrm{O}_{2}$ and evolution of degradation products the performance of the system. Overall, the lower adsorption that the $\beta$-EPI polymer exhibited when $\mathrm{TiO}_{2}$ is bonded to it (Sect. 3.4) together with the lower SMX degradation seem to indicate that this alternative is not recommendable for SMX degradation.

\section{Use of PL for SMX degradation in solution, with and without $\mathrm{H}_{2} \mathrm{O}_{2}$}

The action of PL on SMX free in solution was followed via HPLC (Figs. 7 and 8a). SMX was completely photolyzed by $\mathrm{PL}$, a process that requires $64 \mathrm{~J} / \mathrm{cm}^{2}$. Both by-products appear simultaneously, which indicates at least two degradation pathways.

The addition of $\mathrm{H}_{2} \mathrm{O}_{2}$ to the reaction solution showed no effect on SMX photolysis (Figs. 7 and 8b) when using an $\mathrm{H}_{2} \mathrm{O}_{2} / \mathrm{SMX}$ molar ratio of 25 , except for a reduction in the amount of by-product 2 formed within the first $40 \mathrm{~J} / \mathrm{cm}^{2}$ of fluence applied. It is also possible that SMX is less accessible to hydroxyl radicals when encapsulated into $\beta$-EPI. This effective technique could also be exploited for the photodegradation of residual SMX in solution after the adsorption process (Gómez-Morte et al. 2021).

\section{Conclusion}

$\beta$-EPI polymer is a material useful for the removal of SMX from water, as already tested for other contaminants. It has a low cost compared to other materials such as activated carbons, a good adsorption efficiency, and acts quickly: a contact time lower than $10 \mathrm{~min}$ is enough to reach the adsorption equilibrium. However, the quantitative SMX removal requires the complementary action of AOP, which allows the decomposition of the remains of SMX unadsorbed in solution by photodegradation without the need to add other reactive substances such as hydrogen peroxide.

The main economic and environmental advantage associated with the use of $\beta$-EPI polymer as an adsorbent is its strength and ease with which it can be regenerated in agreement with principles of Circular Economy. In the case of SMX adsorption, the polymer can be rapidly regenerated through the use of hot water at $353 \mathrm{~K}$, obtaining a material that is also more efficient in subsequent adsorption/desorption cycles. As an alternative to desorption, adsorbent material could be regenerated by using advanced oxidation processes (AOP) for the photolysis of adsorbed SMX. PL technology with a fluency of $128 \mathrm{~J} / \mathrm{cm}^{2}$ allows extensive mineralization of SMX. The use of $\beta$-EPI-TiO 2 nanocomposite does not improve the efficiency of AOP, also when 
irradiating the material in continuous UV light, and gives rise to a reduction of the adsorption material capacity.

Acknowledgements The authors wish to thank all who assisted in conducting this work.

Availability of data and materials The datasets used and/or analysed during the current study are available from the corresponding author on reasonable request.

\section{Declarations}

Conflict of interest The authors declare that they have no conflict of interests.

Ethics approval This article does not contain any studies with human partecipants or animal performed by any of the authors.

Open Access This article is licensed under a Creative Commons Attribution 4.0 International License, which permits use, sharing, adaptation, distribution and reproduction in any medium or format, as long as you give appropriate credit to the original author(s) and the source, provide a link to the Creative Commons licence, and indicate if changes were made. The images or other third party material in this article are included in the article's Creative Commons licence, unless indicated otherwise in a credit line to the material. If material is not included in the article's Creative Commons licence and your intended use is not permitted by statutory regulation or exceeds the permitted use, you will need to obtain permission directly from the copyright holder. To view a copy of this licence, visit http://creativecommons.org/licenses/by/4.0/.

\section{References}

Alharbi SK, Kang J, Nghiem LD et al (2017) Photolysis and UV/ $\mathrm{H} 2 \mathrm{O} 2$ of diclofenac, sulfamethoxazole, carbamazepine, and trimethoprim: identification of their major degradation products by ESI-LC-MS and assessment of the toxicity of reaction mixtures. Process Saf Environ Prot 112:222-234. https://doi.org/10.1016/j. psep.2017.07.015

Baranda AB, Lasagabaster A, de Marañón IM (2017) Static and continuous flow-through pulsed light technology for pesticide abatement in water. J Hazard Mater 340:140-151. https://doi.org/10. 1016/j.jhazmat.2017.07.012

Bilal M, Adeel M, Rasheed T et al (2019) Emerging contaminants of high concern and their enzyme-assisted biodegradation - a review. Environ Int 124:336-353

Bizi M (2020) Sulfamethoxazole removal from drinking water by activated carbon: kinetics and diffusion process. Molecules, 25

Bunke D, Moritz S, Brack W et al (2019) Developments in society and implications for emerging pollutants in the aquatic environment. Environ Sci Eur 31:32. https://doi.org/10.1186/ s12302-019-0213-1

Çalışkan E, Göktürk S (2010) Adsorption characteristics of sulfamethoxazole and metronidazole on activated carbon. Sep Sci Technol 45:244-255. https://doi.org/10.1080/01496390903409419
Chong MN, Jin B, Chow CWK, Saint C (2010) Recent developments in photocatalytic water treatment technology: a review. Water Res 44:2997-3027

Deng Y, Zhao R (2015) Advanced Oxidation Processes (AOPs) in wastewater treatment. Curr Pollut Reports 1:167-176. https:// doi.org/10.1007/s40726-015-0015-z

Di Poi C, Costil K, Bouchart V, Halm-Lemeille M-P (2018) Toxicity assessment of five emerging pollutants, alone and in binary or ternary mixtures, towards three aquatic organisms. Environ Sci Pollut Res 25:6122-6134. https://doi.org/10.1007/s11356-017-9306-9

Długosz M, Żmudzki P, Kwiecień A et al (2015) Photocatalytic degradation of sulfamethoxazole in aqueous solution using a floating TiO2-expanded perlite photocatalyst. J Hazard Mater 298:146153. https://doi.org/10.1016/j.jhazmat.2015.05.016

Dulio V, van Bavel B, Brorström-Lundén E et al (2018) Emerging pollutants in the EU: 10 years of NORMAN in support of environmental policies and regulations. Environ Sci Eur 30:5. https:// doi.org/10.1186/s12302-018-0135-3

Garvey M, Rabbitt D, Stocca A, Rowan N (2015) Pulsed ultraviolet light inactivation of Pseudomonas aeruginosa and Staphylococcus aureus biofilms. Water Environ J 29:36-42. https://doi.org/ 10.1111/wej. 12088

Geissen V, Mol H, Klumpp E et al (2015) Emerging pollutants in the environment: a challenge for water resource management. Int Soil Water Conserv Res 3:57-65. https://doi.org/10.1016/j.iswcr.2015. 03.002

Gidwani B, Vyas A (2014) Synthesis, characterization and application of Epichlorohydrin- $\beta$-cyclodextrin polymer. Colloids Surfaces B Biointerfaces 114:130-137

Gómez-López VM, Ragaert P, Debevere J, Devlieghere F (2007) Pulsed light for food decontamination: a review. Trends Food Sci Technol 18:464-473. https://doi.org/10.1016/j.tifs.2007.03.010

Gómez-Morte T, Gómez-López VM, Lucas-Abellán C et al (2021) Removal and toxicity evaluation of a diverse group of drugs from water by a cyclodextrin polymer/pulsed light system. J Hazard Mater 402:123504. https://doi.org/10.1016/j.jhazmat.2020.123504

Hasmath Farzana M, Meenakshi S (2013) Synergistic effect of chitosan and titanium dioxide on the removal of toxic dyes by the photodegradation technique. Ind Eng Chem Res 53:55-63. https://doi. org/10.1021/ie402347g

Kitaoka M, Hayashi K (2002) Adsorption of Bisphenol A by crosslinked $\beta$-cyclodextrin polymer. J Incl Phenom Macrocycl Chem 44:429-431. https://doi.org/10.1023/A:1023024004103

Lam MW, Mabury SA (2005) Photodegradation of the pharmaceuticals atorvastatin, carbamazepine, levofloxacin, and sulfamethoxazole in natural waters. Aquat Sci 67:177-188. https://doi.org/10.1007/ s00027-004-0768-8

Lei M, Zhang L, Lei J et al (2015) Overview of emerging contaminants and associated human health effects. Biomed Res Int 2015:404796. https://doi.org/10.1155/2015/404796

Lin YC, Lee HS (2010) Effects of TiO2 coating dosage and operational parameters on a $\mathrm{TiO} 2 / \mathrm{Ag}$ photocatalysis system for decolorizing Procion red MX-5B. J Hazard Mater 179:462-470. https://doi.org/10.1016/j.jhazmat.2010.03.026

Lopez De Alda MJ, Díaz-Cruz S, Petrovic M, Barceló D (2003) Liquid chromatography-(tandem) mass spectrometry of selected emerging pollutants (steroid sex hormones, drugs and alkylphenolic surfactants) in the aquatic environment. J Chromatogr A 1000:503-526 
Martín J, del Orta M, Medina-Carrasco SM et al (2018) Removal of priority and emerging pollutants from aqueous media by adsorption onto synthetic organo-funtionalized high-charge swelling micas. Environ Res 164:488-494. https://doi.org/10.1016/j. envres.2018.03.037

Martínez-Costa JI, Rivera-Utrilla J, Leyva-Ramos R et al (2018) Individual and simultaneous degradation of the antibiotics sulfamethoxazole and trimethoprim in aqueous solutions by Fenton, Fenton-like and photo-Fenton processes using solar and UV radiations. J Photochem Photobiol A Chem 360:95-108. https:// doi.org/10.1016/j.jphotochem.2018.04.014

Martínez-López S, Lucas-Abellán C, Serrano-Martínez A et al (2019) Pulsed light for a cleaner dyeing industry: Azo dye degradation by an advanced oxidation process driven by pulsed light. J Clean Prod 217:757-766. https://doi.org/10.1016/j.jclep ro.2019.01.230

Min Z (2013) Influence of $\mathrm{TiO}_{2}$ surface properties on water pollution treatment and photocatalytic activity. Bull Korean Chem Soc 34:953-956. https://doi.org/10.5012/BKCS.2013.34.3.953

Moral-Rodríguez AI, Leyva-Ramos R, Ocampo-Pérez R et al (2016) Removal of ronidazole and sulfamethoxazole from water solutions by adsorption on granular activated carbon: equilibrium and intraparticle diffusion mechanisms. Adsorption 22:89-103. https://doi. org/10.1007/s10450-016-9758-0

Morin-Crini N, Winterton P, Fourmentin S et al (2018) Water-insoluble $\beta$-cyclodextrin-epichlorohydrin polymers for removal of pollutants from aqueous solutions by sorption processes using batch studies: a review of inclusion mechanisms. Prog Polym Sci 78:123. https://doi.org/10.1016/j.progpolymsci.2017.07.004

Mourid EH, Lakraimi M, Benaziz L et al (2019) Wastewater treatment test by removal of the sulfamethoxazole antibiotic by a calcined layered double hydroxide. Appl Clay Sci 168:87-95. https://doi. org/10.1016/j.clay.2018.11.005

Murai S, Imajo S, Takasu Y et al (1998) Removal of phthalic acid esters from aqueous solution by inclusion and adsorption on $\beta$-Cyclodextrin. Environ Sci Technol 32:782-787. https://doi. org/10.1021/es970463d

Orprecio R, Evans CH (2003) Polymer-immobilized cyclodextrin trapping of model organic pollutants in flowing water streams. J Appl Polym Sci 90:2103-2110. https://doi.org/10.1002/app.12818

Pellicer JA, Rodríguez-López MI, Fortea MI et al (2019) Adsorption properties of $\beta$ - and Hydroxypropyl- $\beta$-cyclodextrins cross-linked with epichlorohydrin in aqueous solution. Sustain Recycling Strategy Textile Dyeing Process Polymers (Basel). https://doi.org/10. 3390/polym11020252
Peña-Guzmán C, Ulloa-Sánchez S, Mora K et al (2019) Emerging pollutants in the urban water cycle in Latin America: a review of the current literature. J Environ Manage 237:408-423

Prasannamedha G, Kumar PS (2020) A review on contamination and removal of sulfamethoxazole from aqueous solution using cleaner techniques: present and future perspective. J Clean Prod 250:119553. https://doi.org/10.1016/j.jclepro.2019.119553

de Rezende JCT, Ramos VHS, Silva AS et al (2019) Assessment of sulfamethoxazole adsorption capacity on Pirangi clay from the State of Sergipe, Brazil, modified by heating and addition of organic cation. Cerâmica 65:626-634. https://doi.org/10.1590/0366-69132 019653762774

Rizzi V, Gubitosa J, Fini P et al (2020) A “classic" material for capture and detoxification of emergent contaminants for water purification: the case of tetracycline. Environ Technol Innov 19:100812. https://doi.org/10.1016/j.eti.2020.100812

Rizzi V, Gubitosa J, Signorile R et al (2021) Cyclodextrin nanosponges as adsorbent material to remove hazardous pollutants from water: the case of ciprofloxacin. Chem Eng J 411:128514. https://doi.org/ 10.1016/j.cej.2021.128514

Rizzi V, Lacalamita D, Gubitosa J et al (2019) Removal of tetracycline from polluted water by chitosan-olive pomace adsorbing films. Sci Total Environ 693:133620. https://doi.org/10.1016/j.scitotenv. 2019.133620

Rizzi V, Longo A, Placido T et al (2018) A comprehensive investigation of dye-chitosan blended films for green chemistry applications. J Appl Polym Sci 135. https://doi.org/10.1002/app.45945

Rizzi V, Losito I, Ventrella A et al (2015) Rose Bengal-photosensitized oxidation of 4-thiothymidine in aqueous medium: evidence for the reaction of the nucleoside with singlet state oxygen. Phys Chem Chem Phys 17. https://doi.org/10.1039/c5cp03615a

Rizzi V, Mongiovì, ChiaraFini P, Petrella A, et al (2017) Operational parameters affecting the removal and recycling of direct blue industrial dye from wastewater using bleached oil mill waste as alternative adsorbent material. Int J Environ Agric Biotechnol 2:1560-1572. https://doi.org/10.22161/ijeab/2.4.15

Rodil R, Quintana JB, Concha-Graña E et al (2012) Emerging pollutants in sewage, surface and drinking water in Galicia (NW Spain). Chemosphere 86:1040-1049. https://doi.org/10.1016/j. chemosphere.2011.11.053

Rodil R, Quintana JB, López-Mahía P et al (2009) Multi-residue analytical method for the determination of emerging pollutants in water by solid-phase extraction and liquid chromatography-tandem mass spectrometry. J Chromatogr A 1216:2958-2969. https:// doi.org/10.1016/j.chroma.2008.09.041 
Romita R, Rizzi V, Gubitosa J et al (2021) Cyclodextrin polymers and salts: an Eco-Friendly combination to modulate the removal of sulfamethoxazole from water and its release. Chemosphere 283:131238. https://doi.org/10.1016/j.chemosphere.2021.131238

Romita R, Rizzi V, Semeraro P et al (2019) Operational parameters affecting the atrazine removal from water by using cyclodextrin based polymers as efficient adsorbents for cleaner technologies. Environ Technol Innov 16. https://doi.org/10.1016/j.eti.2019.100454

Ryan CC, Tan DT, Arnold WA (2011) Direct and indirect photolysis of sulfamethoxazole and trimethoprim in wastewater treatment plant effluent. Water Res 45:1280-1286. https://doi.org/10.1016/j. watres.2010.10.005

Salazar H, Martins PM, Santos B et al (2020) Photocatalytic and antimicrobial multifunctional nanocomposite membranes for emerging pollutants water treatment applications. Chemosphere 250:126299. https://doi.org/10.1016/j.chemosphere.2020.126299

Serrano-Martínez A, Mercader-Ros MT, Martínez-Alcalá I et al (2020) Degradation and toxicity evaluation of azo dye Direct red $83: 1$ by an advanced oxidation process driven by pulsed light. J Water Process Eng 37:101530. https://doi.org/10.1016/j.jwpe.2020.101530

Sophia AC, Lima EC (2018) Removal of emerging contaminants from the environment by adsorption. Ecotoxicol Environ Saf 150:1-17. https://doi.org/10.1016/j.ecoenv.2017.12.026

Tian B, Hua S, Tian Y, Liu J (2021) Cyclodextrin-based adsorbents for the removal of pollutants from wastewater: a review. Environ Sci Pollut Res 28:1317-1340. https://doi.org/10.1007/ s11356-020-11168-2
Tobajas M, Belver C, Rodriguez JJ (2017) Degradation of emerging pollutants in water under solar irradiation using novel $\mathrm{TiO} 2-\mathrm{ZnO} /$ clay nanoarchitectures. Chem Eng J 309:596-606. https://doi.org/ 10.1016/j.cej.2016.10.002

Trovó AG, Nogueira RFP, Agüera A et al (2009) Photodegradation of sulfamethoxazole in various aqueous media: Persistence, toxicity and photoproducts assessment. Chemosphere 77:1292-1298. https://doi.org/10.1016/j.chemosphere.2009.09.065

Wang W, Saeed A, He J et al (2020) Bio-inspired porous helical carbon fibers with ultrahigh specific surface area for super-efficient removal of sulfamethoxazole from water. J Colloid Interface Sci 578:304-314. https://doi.org/10.1016/j.jcis.2020.05.117

Wols BA, Hofman-Caris CHM (2012) Review of photochemical reaction constants of organic micropollutants required for UV advanced oxidation processes in water. Water Res 46:2815-2827

Yang Y, Lu X, Jiang J et al (2017) Degradation of sulfamethoxazole by UV, UV/H2O2 and UV/persulfate (PDS): Formation of oxidation products and effect of bicarbonate. Water Res 118:196-207. https://doi.org/10.1016/j.watres.2017.03.054

Zheng W, Shi Y, Liu G et al (2020) Heteroatom-doped highly porous carbons prepared by in situ activation for efficient adsorptive removal of sulfamethoxazole. RSC Adv 10:1595-1602. https:// doi.org/10.1039/C9RA09269B 